\title{
ROLE OF OVERLAPPING GENERATIONS IN MAINTAINING GENETIC VARIATION IN A FLUCTUATING ENVIRONMENT
}

\author{
Stephen Ellner* and Nelson G. Hairston, Jr. $\dagger$ \\ * Biomathematics Program, Department of Statistics, North Carolina State University, \\ Raleigh, North Carolina 27695-8203; †Section of Ecology and Systematics, Cornell University, \\ Ithaca, New York 14853-2701
}

Submitted September 17, 1992; Revised April 19, 1993; Accepted May 19, 1993

\begin{abstract}
Population genetics theory suggests that temporally fluctuating selection on phenotypes can act to maintain genetic variance only under very restrictive conditions. However, this conclusion is based on models with discrete nonoverlapping generations. We propose here that temporally fluctuating selection can indeed contribute significantly to the maintenance of genetic variation when the effects of overlapping generations and age-specific or stage-specific selection are considered. We develop a simple model for a population with overlapping generations, experiencing stabilizing selection with a temporally fluctuating optimum, and subject to repeated invasions by mutants with alternative phenotypes. We find that an evolutionarily stable population must have positive genetic variance maintained by selection so long as the product (variance of fluctuations) times (amount of generation overlap) times (selection intensity) is sufficiently high. This result applies to haploid, diploid, single-locus, or multilocus inheritance, and it does not depend on any form of heterozygote advantage to maintain genetic variance. However, it depends on the map between genotype and phenotype being constrained. If a single genotype can produce an arbitrary distribution of phenotypes, then genetic variance is not maintained by selection.
\end{abstract}

Temporally fluctuating selection, in which the relative fitness of different phenotypes varies over time, has repeatedly been proposed and rejected as a significant process maintaining genetic variation in natural populations (Hedrick et al. 1976; Hedrick 1986). Conditions exist under which fluctuations may promote polymorphisms in models for Mendelian (single-locus) traits, but they are typically thought to be too restrictive to be of general importance (Hedrick 1986), with the exception of heterozygote advantages resulting from lower betweengeneration fitness variance as in the SAS-CFF models (Gillespie 1978, 1991). Frank and Slatkin (1990) have described a general scheme that places most formulations of spatially and temporally varying selection on Mendelian traits in a common framework. They conclude that maintenance of a polymorphism by spatially varying selection is likely, whereas under temporally fluctuating selection it is not. Likewise, quantitative genetic models dealing with the maintenance of variation in quantitative (multilocus) traits through mutation-selection balance have shown that random temporal variation in phenotypic fitnesses increases genetic variance not at all, or only slightly, over that maintained under constant stabilizing selection (Lande 1977; Turelli 1988; Barton and Turelli 1989). For

‡ E-mail: S.E., ellner@stat.ncsu.edu; N.G.H., ngh@cornella.cit.cornell.edu. 
haploid models of quantitative trait inheritance, Karlin (1988, p. 143) found that, without any mutation to restore variance, "selection would eliminate genetic variability even when the selection mode varies over successive generations."

Despite the broad consensus of these findings, we propose that temporally fluctuating selection can indeed contribute significantly to the maintenance of genetic variation when the effects of overlapping generations, and age-specific or stage-specific selection, are considered. The mechanism we propose here is quite robust to varied assumptions about the underlying genetic system, applying in both single-locus and multilocus models, and does not depend on any form of heterozygote advantage to maintain genetic variance. The models discussed above make the assumption (prevalent throughout theoretical population genetics) that generations are discrete and nonoverlapping, so our proposal does not conflict with any previous mathematical results. However, it suggests that the biological relevance of many previous results may be limited to organisms with nonoverlapping generations.

Our proposal derives from ecological theory developed to examine the role of temporal environmental variation in interspecific competition, combined with ideas from evolutionary game theory. A number of investigators (e.g., Chesson and Warner 1981; Ellner 1984; Shmida and Ellner 1984; Chesson 1986) have shown that, in an environment that alternates randomly between conditions favorable to one species and those favorable to another, the two species can coexist provided that there is a life-history stage for each that survives over multiple opportunities for reproduction and that is not strongly affected by the features of the environment that vary. Similar results also hold for deterministic variations in conditions affecting reproductive success or population interactions that result in fluctuating population densities (Levin et al. 1977; Levins 1979; Armstrong and McGehee 1980).

The basic result, called the "storage effect" (Chesson 1983; Warner and Chesson 1985), derives from the fact that, although an organism or a population of organisms may experience considerably reduced or even complete loss of recruitment in years favorable to its competitor, it can persist so long as there are life-history stages that are viable for periods long enough to experience the next favorable year. Such life-history stages include long-lived adults (with environmental fluctuations affecting the success of their offspring) and dormant seeds of annual plants (with environmental fluctuations affecting the success of active individuals).

Clearly, in asexual species these ecological models are directly transferable to a genetic system. Coexistence of species in ecological models is equivalent to maintaining a genetically heterogeneous population, so long as the relevant characteristics are genetically determined (Seger and Brockmann 1987). The same situation can also apply to models of sexual diploid species (see, e.g., Haldane and Jayakar 1963; Karlin and Lieberman 1974). Significantly for our analogy with genetic polymorphism, both Chesson (1984) and Seger and Brockmann (1987) have shown that temporal variation is roughly comparable to spatial variation in maintaining diversity in long-lived organisms with overlapping generations.

However, the situation described by these two-species ecological models and the analogous two-allele genetic models is essentially a "protected polymor- 
phism" maintained by balancing selection among a few particular types, none of which may be at or near the optimal phenotype for the selection regime. Thus, these models do not address the outcome of natural selection acting on a population containing a continuous range of phenotypes for the trait of interest. A wellknown feature of evolution in fluctuating environments is the expectation that individuals should evolve a single phenotype, presumably supported by a single genotype (see, e.g., Cohen 1966; Slatkin 1974; Seger and Brockmann 1987; Philippi and Seger 1989). This bet-hedging strategy is predicted to exclude all others because, although it never (or virtually never) performs better than all other types in a given year, it never performs really badly and is best overall in the long run.

Therefore, our main goal here is to explore theoretically the conditions under which a genetically variable population is evolutionarily stable. Evolutionary stability is defined by the property that any rare mutant not already present in the population will be removed by selection (Maynard Smith 1982). The significance of examining evolutionary stability in order to identify conditions under which genetic variance is maintained is illustrated by models of seed dormancy strategies (Ellner 1985). As in Seger and Brockmann's (1987) model, there are pairs of strategies that will coexist in a fluctuating environment (i.e., both are persistent in the sense of stochastic boundedness; see Chesson and Ellner 1989). However, there is always a single evolutionarily stable strategy (ESS) that outcompetes and in the long run excludes all other strategies. Unless the ESS strategy can only be achieved by a heterozygote, selection does not maintain genetic variance.

We develop our proposal by analyzing a simple model for fluctuating selection with overlapping generations. The model derives from consideration of the effects of long-term egg diapause on the maintenance of genetic variation in a population of freshwater copepods (planktonic crustaceans), Diaptomus sanguineus, exposed to annual variations in the direction and intensity of selection imposed by fish predation (Hairston 1988; Hairston and Dillon 1990). The model is a generalization of the processes operating in that specific example that is applicable to a broad variety of circumstances. A key feature of the model is the presence of a life-history stage immune to the environmental fluctuations, which allows the storage effect to operate. Consequently, more risk-prone types that have done poorly in recent years are quickly reestablished and may take advantage of favorable conditions when they occur. When the immune stage provides an adequate safety net and environmental variability is high, a bet hedger is no longer able to exclude all of the more risk-prone strategies.

Our second goal is to explore the behavior in this system of a second type of bet hedger: the mixed strategy. We generalize Seger and Brockmann's (1987) proposal that, if it is possible for a single genotype to produce multiple phenotypes, then there will be some ESS mixture of phenotypes that can successfully invade any population of single-phenotype genotypes.

\section{A MODEL}

Immediately before the reproductive season each year, the population under consideration consists entirely of individuals in the persistent stage of the stage of life cycle (e.g., diapausing eggs or long-lived adults). Let $X_{i}(t)$ be the abundance 
of type $i$ individuals (or females, in a sexual population) in year $t$; we assume for now that "type" is both phenotype and genotype (haploid genetics without mutation) and ignore age structure within the persistent stage. A fraction $H>0$ of the type $i$ population engages in reproduction, with a per capita fecundity $Y_{i}(t)$ and per capita survival $s_{\mathrm{r}}<1$. The reproductively inactive ("dormant") individuals survive at per capita rate $0<s_{\mathrm{d}}<1$. All types are assumed to have the same $H, s_{\mathrm{r}}$, and $s_{\mathrm{d}}$. Define $\gamma=H s_{\mathrm{r}}+(1-H) s_{\mathrm{d}}$, so that $\gamma$ measures the amount of generational overlap. The population dynamics are then (Cohen 1966)

$$
X_{i}(t+1)=X_{i}(t) *\left[H Y_{i}(t)+\gamma\right] .
$$

If diapausing eggs or seeds are the persistent stage, then $H$ is the fraction hatching or germinating each year and $s_{\mathrm{r}}=0$, so that $\gamma=(1-H) s_{\mathrm{d}}$.

Selection occurs because the trait of interest is assumed to affect the fecundity (number of surviving offspring) of active individuals. That is, $Y_{i}(t)$ is phenotypedependent. We assume that phenotypes can be described by a single variable $D$ and that the relative fitness in a given year $(R)$ depends on the deviation between type $i$ 's phenotype $\left(D_{i}\right)$ and the optimal phenotype in year $t\left(M_{t}\right)$. An example is Gaussian stabilizing selection with a fluctuating optimum, in which the relative fitness of type $i$ in year $t$ is

$$
R\left(D_{i}-M_{t}\right)=\exp \left[-\left(D_{i}-M_{t}\right)^{2} / 2 \sigma_{w}^{2}\right] .
$$

The intensity of selection in equation (2) is determined by $\sigma_{w}$, the standard deviation of the Gaussian curve describing relative fitnesses, with more intense selection if $\sigma_{w}$ is small. In general, we assume that deviations from the optimal phenotype are penalized-that is, that $R(x)>R(y)$ if $0<x<y$ or $y<x<0$. "Environmental variance" (phenotypic variance not under genetic control) can be accommodated within this model by replacing $R\left(D_{i}-M_{t}\right)$ by the average of $R\left(D-M_{t}\right)$ over the phenotypic distribution of type $i$ individuals.

To specify the absolute fitnesses we use the "saturating yield" model introduced by Levin et al. (1984); that is, we assume that the total number of offspring produced in a given year by all types combined is a constant $K>0$. This implies that

$$
Y_{i}(t)=\frac{K R\left(D_{i}-M_{t}\right)}{\sum_{j} H X_{j}(t) R\left(D_{j}-M_{t}\right)},
$$

where $R$ is the relative fitness function, and the index $j$ runs over all types in the population. Since the total offspring production is always $K$, it follows from equation (1) that

$$
\sum_{j} X_{j}(t+1)=K+\gamma \sum_{j} X_{j}(t) .
$$

Consequently, the total population size converges to the stable equilibrium

$$
\bar{X}=K /(1-\gamma) \text {. }
$$


Thus, selection is frequency-dependent but not density-dependent; and when there is only one type in the population, its abundance converges to $\bar{X}$. Experience with other models (Levin et al. 1984; Ellner 1985; Cohen and Levin 1991) suggests that results obtained for the saturating yield model generalize qualitatively to some more realistic models of density-dependent competition. Except for very low total densities such that the denominator of expression (3) is not much greater than one, the saturating yield model will be nearly equivalent to the equation used in an earlier article (Hairston and Munns 1984) to model fecundity in Diaptomus sanguineus. Another motivation for this model is the "law of constant final yield" for plant populations limited by crowding (Harper 1977). When this law applies, the saturating yield model is equivalent to assuming that seed output is a constant fraction of total yield.

\section{INVASIBILITY ANALYSIS AND EVOLUTIONARY STABILITY}

Whether coexistence occurs in infinite-population stochastic models such as ours usually can be determined from an invasibility analysis based on the boundary growth rate of each competitor (Turelli 1978; Chesson and Warner 1981). For types $d$ and $D$, type $d$ 's boundary growth rate, $\rho(d, D)$, is essentially defined to be the log of the geometric mean growth rate for a small type $d$ subpopulation invading an established population of type $D$ individuals. Consequently,

$$
\begin{aligned}
& \text { If } \rho(d, D)<0 \text {, then } d \text { cannot invade } D ; \\
& \text { If } \rho(d, D)>0 \text { and } \rho(D, d)>0 \text {, then } d \text { and } D \text { will coexist. }
\end{aligned}
$$

In our model, from equation (1) we have $\rho(d, D)=E \ln [H Y(d, D, t)+\gamma]$ where $Y(d, D, t)$ is the per capita fecundity of rare type $d$ eggs in a stable population of type $D$ individuals; that is,

$$
Y(d, D, t)=\frac{K R\left(d-M_{t}\right)}{H \bar{X} R\left(D-M_{t}\right)}=(1-\gamma) / H \frac{R\left(d-M_{t}\right)}{R\left(D-M_{t}\right)} .
$$

Therefore, in our model

$$
\rho(d, D)=E \ln \left[(1-\gamma) \frac{R\left(d-M_{t}\right)}{R\left(D-M_{t}\right)}+\gamma\right] .
$$

Previous articles (Chesson and Ellner 1989; Ellner 1989) give the general definition of boundary growth rates and technical conditions under which the invasibility analysis outlined above is valid. For the specific examples discussed later, those conditions are satisfied. Therefore, if a type $D^{*}$ is an ESS, it must be the case that $\rho\left(d, D^{*}\right) \leq 0$ for all $d \neq D^{*}$ and hence that

$$
\frac{\partial \rho}{\partial d}\left(D^{*}, D^{*}\right)=0
$$

(the first-order condition for a local maximum). We will refer to any solution of equation (7) as a "candidate ESS."

Our goal in analyzing the model is to determine whether a population containing 
multiple types will tend to become monomorphic or instead will settle into fluctuations with many types coexisting. We use Ludwig and Levin's (1991) approach based on a Taylor series approximation to $\rho(d, D)$ near a candidate ESS $D^{*}$; a second-order approximation is necessary because all first-order terms vanish at a candidate ESS. Define

$$
\rho_{d d}=\frac{\partial^{2} \rho}{\partial d^{2}}\left(D^{*}, D^{*}\right), \quad \rho_{D D}=\frac{\partial^{2} \rho}{\partial D^{2}}\left(D^{*}, D^{*}\right) .
$$

The signs of $\rho_{D D}$ and $\rho_{d d}$ determine the sign of $\rho\left(D^{*}, d\right)$ and $\rho\left(d, D^{*}\right)$, respectively, for $d$ and $D$ near $D^{*}$. These tell us whether $D^{*}$ can invade and be invaded by nearby types (i.e., types other than $D^{*}$ in some interval around $D^{*}$ ). Specifically, if $\rho_{d d}>0$, then $D^{*}$ can be invaded by any nearby type; but if $\rho_{d d}<0$, then $D^{*}$ is a local ESS that cannot be invaded by any nearby types. If $\rho_{D D}>0$, then $D^{*}$ can invade all nearby types; but if $\rho_{D D}<0$, then $D^{*}$ cannot invade any nearby types.

Two cases are particularly important for our analysis. If $\rho_{d d}<0$ and $\rho_{D D}>0$, then $D^{*}$ is noninvasible and can itself invade any nearby strategy; hence, $D^{*}$ is a continuously stable strategy, or CSS (Eshel and Motro 1981; Eshel 1983). In this case a monomorphic population with all individuals having a CSS phenotype would be the predicted outcome of natural selection. On the other hand, if $\rho_{d d}>$ 0 for all candidate ESSs, then any monomorphic population is invasible, and multiple types must be coexisting at any stable state of the population. A candidate ESS with $\rho_{d d}>0$ and $\rho_{D D}>0$ can be invaded by nearby types, but it can itself invade all nearby types; $D^{*}$ is then an evolutionarily compatible strategy, or ECS, as defined by Cohen and Levin (1991).

\section{RESULTS}

The conclusion from invasibility analysis is that whether a genetically variable population is evolutionarily stable in our model depends on the signs of $\rho_{d d}$ and $\rho_{D D}$. For our model it is straightforward to calculate these quantities in terms of the relative fitness function $R$, by differentiating inside the expectation in equation (6). This requires some mild assumptions about the selection regime; it is sufficient to assume that the optimal phenotype $M_{t}$ lies within some finite interval $\left[m_{1}\right.$, $m_{2}$ ] with probability 1 and that $R$ and $1 / R$ are twice continuously differentiable on $\left[m_{1}, m_{2}\right]$.

For convenience in presenting the results from these calculations, we first scale $R$ so that $R(0)=1$; this can always be done since $R$ measures relative rather than absolute fitnesses. We then define $\phi(x)$ by $R(x)=\exp (-b \cdot \phi(x))$, where $\phi$ is a nonnegative function with $\phi(0)=0$ and $\phi(1)=1$. Then $b \cdot \phi\left(D_{i}-M_{t}\right)$ is a measure of the penalty for deviating from the optimal phenotype in year $t$, and the parameter $b$ measures the intensity of selection. In terms of $b$ and $\phi$, the condition (7) defining a candidate ESS can be expressed as

$$
E\left[\phi^{\prime}\left(D^{*}-M_{t}\right)\right]=0,
$$

and we have

$$
\rho_{d d}=C \cdot(\gamma b \tilde{V}-\tilde{E})
$$


and

$$
\rho_{D D}=C \cdot(\gamma b \tilde{V}+\tilde{E}),
$$

where

$$
\begin{gathered}
\tilde{V}=\operatorname{var}\left[\phi^{\prime}\left(D^{*}-M_{t}\right)\right], \\
\tilde{E}=E\left[\phi^{\prime \prime}\left(D^{*}-M_{t}\right)\right],
\end{gathered}
$$

and $C$ is a positive number whose value depends on model parameters.

The general conclusion from equation (8) is that overlapping generations promotes the maintenance of genetic variation under fluctuating selection. Note that $\tilde{V}$ is nonnegative, and, if the optimal phenotype $M_{t}$ fluctuates, then $\tilde{V}$ will be positive. An increase in the amount of generational overlap $\gamma$ (e.g., by increasing the survival rate $s_{\mathrm{r}}$ or $s_{\mathrm{d}}$ ) has no effect on the values of $D^{*}, \tilde{E}$, or $\tilde{V}$. Hence, such changes may move $\rho_{d d}$ from negative (in which case $D^{*}$ is an ESS) to positive ( $D^{*}$ can be invaded), but not vice versa. Thus, the general effect of generational overlap is to destabilize any population equilibrium at which the genetic variance is zero.

For Gaussian stabilizing selection (eq. [2]), $\phi(x)=x^{2}$ and $b=1 /\left(2 \sigma_{w}^{2}\right)$. The only candidate ESS is $D^{*}=E\left(M_{t}\right)$, the optimal phenotype under average conditions, so we have $\tilde{E}=2$ and $\tilde{V}=4 \operatorname{var}\left(M_{t}\right)$. Therefore $\rho_{D D}>0$, so $D^{*}$ is either a CSS or an ECS, depending on whether $\rho_{d d}$ is negative or positive. Substituting the values of $\tilde{V}$ and $\tilde{E}$ into expression (8) and using the definition of $b$ give that $D^{*}$ is an ECS if

$$
\gamma \operatorname{var}\left(M_{t}\right) / \sigma_{w}^{2}>1
$$

and $D^{*}$ is a CSS if equation (9) holds with the sign reversed. When equation (9) holds, there is no ESS, so as noted earlier a population with zero genetic variance cannot be evolutionarily stable.

Consequently, expression (9) is the condition under which genetic variance is maintained at evolutionary equilibrium with Gaussian stabilizing selection. An intuitive explanation of equation (9) is as follows. Suppose that the persistent stage consists of diapausing eggs. If generations do not overlap $(H=1)$, the winning strategy is to adopt the phenotype with the highest fitness in the average environment. But if eggs can persist in dormancy and the temporal environmental variability is sufficiently high, the phenotype that "bets" on the average can be invaded by but also can invade riskier phenotypes that have more widely fluctuating fitnesses and rely on the egg bank to cushion their losses in bad years. Similarly, a short-lived adult with few opportunities to reproduce must bet on the average, while a long-lived adult can play a riskier strategy with occasional big payoffs.

These qualitative results do not depend on selection being exactly Gaussian. One simple generalization is to consider fitness penalties of the form $\phi(x)=$ $\psi(|x|)$, where $\psi^{\prime}(x)>0$ and $\psi^{\prime \prime}(x)>0$ for $x>0$. Then $\tilde{E}$ and $\tilde{V}$ are both positive, so as with Gaussian selection any candidate ESS is either a CSS or an ECS. The condition for maintaining genetic variance is $\rho_{d d}>0$, which is true if

$$
\gamma b>\tilde{E} / \tilde{V} \text {. }
$$


Thus, genetic variance will be maintained if selection is intense and there is a high level of generational overlap, and genetic variance will be lost if selection is too weak or there is too little overlap.

If $\phi^{\prime \prime}$ is not always positive, then it is possible to have $\tilde{E} \leq 0$. In such cases $\rho_{d d}$ is positive, so long as the phenotypic optimum fluctuates and there is some longterm dormancy. Hence, any candidate ESS is invasible, and genetic variance will be maintained.

Two simple but significant extensions of these results are possible. First, the conditions for maintaining genetic variance can be extended in the usual way to diploid models with an arbitrary finite number of loci, under the standard assumptions of random mating and infinite population size. Assume that the trait of interest depends on the individual's genotype at a single locus with incomplete dominance. The dynamics of a phenotypically monomorphic population in our model are independent of the genetics. When a rare mutant allele enters such a population, it is present almost exclusively in individuals heterozygous at the locus where the mutation arose. Consequently, the boundary growth rate (eq. [5]) is correct for invasion by a rare allele such that heterozygotes are type $d$, whereas homozygotes for the common allele are type $D$. It therefore follows that if type $D^{*}$ is an ECS as defined earlier, any genetically homogeneous population can be invaded by rare alleles. Conversely, if $D^{*}$ is an ESS and there is an allele $A$ such that $A A$ homozygotes are type $D^{*}$, then all rare alleles will have negative boundary growth rates and cannot invade.

The second extension is to allow fluctuations in the intensity of selection, for example, by changing the relative fitness function to $R\left(d_{i}, t\right)=\exp \left(-I_{t} b \phi\left[d_{i}-\right.\right.$ $\left.M_{t}\right]$ ) where $I_{t}$ is a nonnegative random variable with a mean of one, representing fluctuations in selection intensity. If $I_{t}$ and $M_{t}$ are independent, the value of $\tilde{V}$ is increased by a factor of $1+\operatorname{var}\left(I_{t}\right)$, and otherwise equation (8) and the conditions for maintaining genetic variance are unchanged. Thus, fluctuations in selection intensity that are uncorrelated with fluctuations in the optimal phenotype favor the maintenance of genetic variance. If $I_{t}$ and $M_{t}$ are not independent, equation (10) still holds (with slightly different formulas for $\tilde{V}$ and $\tilde{E}$ ), so fluctuations in selection intensity do not affect the conclusion that genetic variance is maintained when there is strong selection and a high level of generational overlap.

\section{SIMULATIONS}

The invasibility analysis only considers pairwise competition, and it is local (i.e., it applies for phenotypes near a candidate ESS). Extrapolation of these results to competition among many types, some of them far from a candidate ESS, is a reasonable but unproved conjecture. We therefore ran simulations of the model to observe its behavior over an extended period and verify the conjectures that an ESS also outcompetes any "coalition" of invaders and that the existence of an ECS implies that the population tends to develop an evolutionarily stable combination, or ESC (Ludwig and Levin 1991), of several coexisting types.

Baseline values of parameters were chosen in rough correspondence with the situation occurring in the copepod population mentioned earlier. The character under selection is the date at which females switch to producing diapausing eggs 
rather than immediately hatching eggs. The interannual variation in the optimal switch date is Gaussian with a standard deviation of $13 \mathrm{~d}$ (Hairston and Munns 1984; Hairston 1988). Gaussian stabilizing selection was employed with selection intensity set at $b=0.01$ to approximate the selection intensities estimated from a cohort model of the population dynamics (Braner and Hairston 1989). The fraction of eggs deposited in the lake sediment that emerge from diapause in any given year is unknown but proposed to be less than 50\% (Hairston and De Stasio 1988). Based on De Stasio (1989) we used $H=0.25$. Survival of dormant eggs is unknown but presumed to be high: $s_{\mathrm{d}}=0.9$.

Results from our simulations are fully consistent with equation (9). The baseline parameters are in the ECS region, and a positive variance is maintained (fig. $1 A$ ), which is similar to typical phenotypic variances observed in the copepod population (median phenotypic SD $=8.5 \mathrm{~d}, n=9$; Hairston and Dillon 1990). Reducing the standard deviation of the phenotypic optimum $M_{t}$ to $7 \mathrm{~d}$ or increasing $H$ to 0.85 moves the model into the ESS region, and the phenotypic variance drops toward zero (figs. $1 B, C$ ). Over 100 other parameter combinations were tried, with parameters chosen and simulations run by students as a homework exercise, and the results were again consistent with equation (9).

These results underscore the significance of using evolutionary stability, rather than the existence of alleles that can be maintained in a polymorphism, as the mathematical criterion for identifying conditions under which selection maintains genetic variance. For all of the parameter values shown in figure 1, there are many pairs of alleles that could coexist in a two-allele polymorphism maintained by selection (this can be proved by approximating $\rho$ near $D^{*}$ as discussed previously and finding types that can invade each other). An analysis based on evolutionary stability allows us to identify the differences in the long-term dynamics.

\section{MIXED-STRATEGY BET HEDGING}

We can now explore, much as Seger and Brockmann (1987) have done, what role a mixed strategy plays in our previous result. The dynamics of our haploid model in equation (1) can be expressed as

$$
X_{i}(t+1)=X_{i}(t) r_{i}(t)
$$

where

$$
r_{i}(t)=H Y_{i}(t)+\gamma .
$$

If we assume that there is a stable combination and choose any two members $i$ and $j$, then the long-term average growth of each type is zero:

$$
E\left[\log r_{i}(t)\right]=E\left[\log r_{j}(t)\right]=0 .
$$

Consider invasion of the combination by a mixed strategy in which each individual chooses phenotype $i$ with probability $p$ and phenotype $j$ with probability 1 $p$. The mixed strategy's boundary growth rate is then

$$
E\left\{\log \left[p r_{i}(t)+(1-p) r_{j}(t)\right]\right\} .
$$

By Jensen's inequality (Breiman 1992), unless $r_{i}(t)=r_{j}(t)$ with probability 1, 


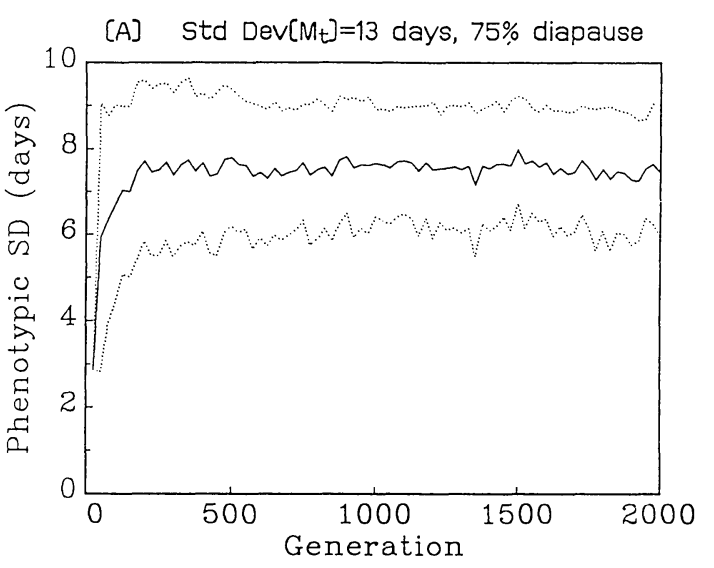

A

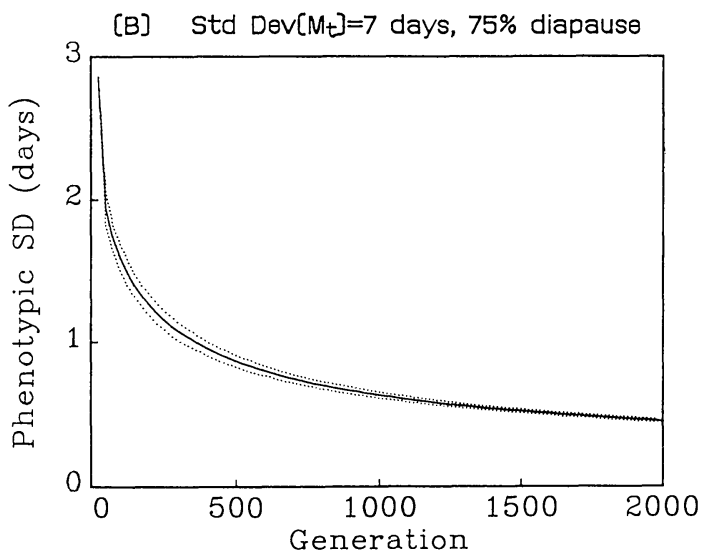

B



C 
equation (12) is always greater than

$$
E\left[p \log r_{i}(t)+(1-p) \log r_{j}(t)\right],
$$

which equals zero by equation (11). Thus, if mixed strategies are possible, any combination containing several distinct genotypes is invasible. This result also applies if the combination consists of mixed strategies; hence, if all mixed strategies are possible, there cannot be an evolutionarily stable combination. However, the advantage of mixed strategies depends on the occurrence of environmental variability: if fitnesses are constant, then equation (11) implies that $r_{i}(t) \equiv r_{j}(t) \equiv$ 1 , so a mixed strategy does not confer increased fitness.

\section{CONCLUSIONS}

Organisms with life histories that dictate strongly overlapping generations have the capacity to maintain genetic variation in temporally fluctuating environments. It is the storage of genotypes in long-lived stages on which the fluctuating selection does not act that provides the mechanism for maintenance of polymorphism. Given sufficient levels of generational overlap, environmental variation, or both, genetic and phenotypic variation is maintained. The appearance of a genotype that can produce a mixture of phenotypes leads in theory to maintenance of phenotypic variation but the loss of genetic variation. The latter result is not unique to our model. Indeed, as Gillespie and Turelli (1989) observe, the maintenance of genetic variation by selection always depends on constraints to what a single genotype can achieve: if a model contains a genotype that is best under all conditions, then genetic variance cannot be maintained by selection. In Cohen's (1970) model for the optimal timing of diapause, mixed strategies were generally favored under density-independent selection with nonoverlapping generations. However, Ludwig and Levin (1991) found that coexistence of mixed strategies could occur in a spatially structured model for plant populations with nonoverlapping generations. Consequently, our result that mixed strategies cannot coexist may not persist in models with age or spatial structure.

We have obtained general results by restricting our analysis to the qualitative question of whether selection on phenotypes acts to remove variance (the ESS/ CSS case) or maintain variance (the ECS case). Our presumption, in interpreting the results, is that these would generally correspond to "low" versus "high" levels of variation in realistic settings where mutation and drift are also operating.

FIG. 1.-Dynamics of the frequency distribution of phenotypes in the haploid model (eq. [1]), with Gaussian stabilizing selection (eq. [2]) and parameters based on Diaptomus sanguineus populations in Rhode Island. Each plot is based on 100 simulation runs of the model with the same parameter values and initial conditions but different random fluctuations in selection. Solid curves show the mean (over simulation runs) of the phenotypic frequency distribution's standard deviation; dotted curves show the mean \pm 1 SD (over simulation runs) of the same quantity. In all simulations the survival rate of dormant eggs was $s=0.9$, the selection intensity was $b=1 /\left(2 \sigma_{w}^{2}\right)=0.01$, and the distribution of the optimal phenotype $M_{t}$ was a zero mean Gaussian. $A, H=0.25, \mathrm{SD}\left(M_{t}\right)=13$ d. $B, H=0.25, \operatorname{SD}\left(M_{t}\right)=7 \mathrm{~d}$. $C, H=0.85, \operatorname{SD}\left(M_{t}\right)=13 \mathrm{~d}$. 
However, further work is necessary to determine how strongly the specific genetic basis of a trait can affect the equilibrium level of phenotypic variance under a given selective regime in the ECS case.

For the case of haploid asexual inheritance, our model exemplifies the fact that overlapping generations allow fluctuating selection to maintain a genetic polymorphism. Previously this situation was generally thought to be impossible (J. Gillespie, personal communication), although the result is closely related to some recent work in population genetics, and the corresponding result for competing species has been in the literature for over a decade (Chesson and Warner 1981). Frank and Slatkin (1990) observe that standard models for haploid selection fail to maintain polymorphism because all individuals of the same genotype have the same fitness, and they mention that spatial heterogeneity could create fitness variations that allow polymorphism. Our results show that, as one might expect, overlapping generations can have the same effect. Gillespie (1991, pp. 154-189) studies the dynamics of " $c$-haploid" models via diffusion approximations, including the result that polymorphism can occur for $c<1$. The $c$-haploid model is a standard discrete-generation haploid model in which the change in allele frequencies between generations is multiplied by an arbitrary constant $c>0$. Gillespie (1991) uses $c$-haploid models strictly as a tool for analyzing diploid models. However, it is easy to show (by converting to allele frequencies rather than numbers) that, in the case of haploid inheritance, our model is equivalent to a $c$-haploid model with $c=(1-\gamma)$.

Several other theoretical studies have suggested that environmental variability can contribute to maintaining genetic variability, but the mechanisms are very different from the one proposed here. One mechanism is a lower fitness variance in heterozygotes relative to homozygotes. Gillespie's SAS-CFF models (Gillespie $1978,1991)$ incorporate genotype-environment interactions such that in each environmental state the fitness of the heterozygote is at least the arithmetic mean of the homozygote fitnesses, which allows polymorphisms to be maintained. Similarly, Gillespie and Turelli (1989) showed that genotype-environment interactions could maintain additive genetic variance in a polygenic model without fluctuating selection. In their model alleles have additive effects on the phenotype, so a genotype's variance in fitness over environments is a decreasing function of the number of heterozygous loci, and consequently polymorphisms are maintained by selection. A. Kondrashov (personal communication) has found that fluctuating selection with large fluctuations in the optimal phenotype can increase the amount of genetic variance maintained by mutation-selection balance, in a polygenic model similar to that of Turelli (1988). In Kondrashov's model generations do not overlap, and without mutation all genetic variance would be lost.

In the copepod example referred to earlier, the authors (Hairston and Munns 1984) found substantial phenotypic variation in an important fitness trait (reproductive phenology) expressed in a population of freshwater copepods known to experience significant interannual variation in the direction and intensity of selection (Hairston 1988). A subsequent article (Hairston and Dillon 1990) reported that, in both field and laboratory studies, a substantial fraction of the observed phenotypic variation was genetically based. They raised the question of whether 
the presence of an egg bank of propagules in long-term dormancy (shown to exist in Hairston and De Stasio 1988; De Stasio 1989), in combination with fluctuating selection, might furnish an explanation for the existence of genetic variation where none might otherwise have been expected.

The results presented here provide a general foundation in support of such an interpretation but with an intriguing caveat: genetic variation is expected, but only so long as a mixed-strategy bet hedger is absent. It is difficult without additional information to proceed further in comparing our theoretical and empirical results. Obviously, for any specific system such as the copepod population that motivated our exploration, more data and more detailed models are needed. At present, we can only raise but not answer a number of questions. Is a mixed-strategy bet hedger truly absent from the copepod population? If so, is mixed-strategy bet hedging prevented by some constraint against it such as physiological cost or inherent complexity of the proposed adaptation? If not, is trait heritability explained by the presence of more than one mixed-strategy genotype, each producing a different mixture of phenotypes?

Overlapping generations are not special cases in the biological world. Longterm dormancy is widespread among organisms in all five kingdoms, including a wide variety of microorganisms (Henis 1987), algae (Sandgren 1988), higher plants (Leck et al. 1989), aquatic invertebrates (e.g., rotifers: Nipkow 1961; crustaceans: Hutchinson 1967; and probably a large number of freshwater and marine invertebrates with dormant stages), and insects with prolonged dormancy (Tauber et al. 1986). An equally large array of organisms have long-lived, iteroparous adults. One group of these, coral reef fishes, was the topic of an early article on the storage effect and species diversity in ecological communities (Chesson and Warner 1981). There are, indeed, many organisms that have both dormant propagules and long-lived adults (e.g., perennial plants).

Overlapping generations, especially plant seed banks, have for the most part been viewed as phenomena slowing down the rate of evolutionary change (Templeton and Levin 1979; Hairston and De Stasio 1988; Venable 1989). It is in some ways paradoxical that the same phenomena should be responsible for the maintenance of genetic variation on which evolutionary response to natural selection depends. Yet it is precisely because genetic change is slow in long-lived stages immune to selection that variation is maintained. Populations with high genetic variation are then capable of significant changes in gene frequencies in response to short-term changes in selection. In the face of sustained directional selection, however, response is limited by the rate of removal of variation from the pool of genotypes stored in long-lived individuals.

\section{ACKNOWLEDGMENTS}

We thank E. Leigh for early on goading us into thinking about the problem of genetic variation and the storage effect and S. Levin, D. Ludwig, J. Gillespie, A. Sasaki, M. Slatkin, M. Turelli, and the editors for comments on drafts of the manuscript. This research was supported by National Science Foundation grant DEB-9118894 to S.E. and N.G.H. 


\section{LITERATURE CITED}

Armstrong, R. A., and R. McGehee. 1980. Competitive exclusion. American Naturalist 115:151-170.

Barton, N. H., and M. Turelli. 1989. Evolutionary quantitative genetics: how little do we know? Annual Review of Genetics 23:337-370.

Braner, M., and N. G. Hairston, Jr. 1989. From cohort data to life table parameters via stochastic modeling. Pages 81-92 in L. McDonald, B. Manly, J. Lockwood, and J. Logan, eds. Estimation and analysis of insect populations. Lecture Notes in Statistics Vol. 55. Springer, Berlin.

Breiman, L. 1992. Probability. Society for Industrial and Applied Mathematics, Philadelphia.

Chesson, P. L. 1983. Coexistence of competitors in a stochastic environment: the storage effect. Lecture Notes in Biomathematics 54:188-198.

1984. The storage effect in stochastic population models. Lecture Notes in Biomathematics 54:76-89.

1986. Environmental variation and the coexistence of species. Pages $240-256$ in T. Case and J. Diamond, eds. Community ecology. Harper \& Row, New York.

Chesson, P. L., and S. Ellner. 1989. Invasibility and stochastic boundedness in monotonic competition models. Journal of Mathematical Biology 27:117-138.

Chesson, P. L., and R. R. Warner. 1981. Environmental variability promotes coexistence in lottery competitive systems. American Naturalist 117:923-943.

Cohen, D. 1966. Optimizing reproduction in a randomly varying environment. Journal of Theoretical Biology 12:119-129.

1970. A theoretical model for the optimal timing of diapause. American Naturalist 104: 389-400.

Cohen, D., and S. A. Levin. 1991. Dispersal in patchy environments: the effects of temporal and spatial structure. Theoretical Population Biology 39:63-99.

De Stasio, B. T. 1989. The seed bank of a freshwater crustacean: copepodology for the plant ecologist. Ecology 70:1377-1389.

Ellner, S. 1984. Asymptotic behavior of some stochastic difference equation population models. Journal of Mathematical Biology 19:169-200.

1985. ESS germination strategies in randomly varying environments. I. Logistic-type models. Theoretical Population Biology 28:50-79.

1989. Convergence to stationary distributions in two species stochastic competition models. Journal of Mathematical Biology 27:451-462.

Eshel, I. 1983. Evolutionary and continuous stability. Journal of Theoretical Biology 103:99-111.

Eshel, I., and U. Motro. 1981. Kin selection and strong evolutionary stability of mútual help. Theoretical Population Biology 19:420-433.

Frank, S. A., and M. Slatkin. 1990. Evolution in a variable environment. American Naturalist 136: 244-260.

Gillespie, J. H. 1978. A general model to account for enzyme variation in natural populations. V. The SAS-CFF model. Theoretical Population Biology 14:1-45.

1991. The causes of molecular evolution. Oxford University Press, Oxford.

Gillespie, J. H., and M. Turelli. 1989. Genotype-environment interactions and the maintenance of polygenic variation. Genetics 121:129-139.

Hairston, N. G., Jr. 1987. Diapause as a predator avoidance adaptation. Pages 281-290 in W. C. Kerfoot and A. Sih, eds. Predation: direct and indirect impacts on aquatic communities. New England University Press, Hanover, N.H.

. 1988. Interannual variation in seasonal predation: its origin and ecological importance. Limnology and Oceanography 33:1245-1253.

Hairston, N. G., Jr., and B. T. De Stasio, Jr. 1988. Rate of evolution slowed by a dormant propagule pool. Nature (London) 336:239-242.

Hairston, N. G., Jr., and T. A. Dillon. 1990. Fluctuating selection and response in a population of freshwater copepods. Evolution 44:1796-1805.

Hairston, N. G., Jr., and W. R. Munns, Jr. 1984. The timing of copepod diapause as an evolutionarily stable strategy. American Naturalist 123:733-751. 
Haldane, J. B. S., and S. D. Jayakar. 1963. Polymorphism due to selection in varying directions. Journal of Genetics 58:237-242.

Harper, J. 1977. Population biology of plants. Academic Press, New York.

Hedrick, P. W. 1986. Genetic polymorphism in heterogeneous environments: a decade later. Annual Review of Ecology and Systematics 17:535-566.

Hedrick, P. W., M. E. Ginevan, and E. P. Ewing. 1976. Genetic polymorphism in heterogeneous environments. Annual Review of Ecology and Systematics 7:1-33.

Henis, Y. 1987. Survival and dormancy of microorganisms. Wiley-Interscience, New York.

Hutchinson, G. E. 1967. A treatise on limnology. II. Introduction to lake biology and limnoplankton. Wiley, New York.

Karlin, S. 1988. Non-Gaussian phenotypic models of quantitative traits. Pages 123-144 in E. J. Eisen, M. M. Goodman, G. Namkoong, and B. S. Weir, eds. The Second International Conference on Quantitative Genetics. Sinauer, Boston.

Karlin, S., and U. Lieberman. 1974. Random temporal variation in selection intensity: case of large population size. Theoretical Population Biology 6:355-382.

Lande, R. 1977. The influence of the mating system on the maintenance of genetic variability in polygenic characters. Genetics 86:485-498.

Leck, M. A., V. T. Parker, and R. L. Simpson, eds. 1989. Ecology of soil seed banks. Academic Press, New York.

Levin, B. R., F. M. Stewart, and L. Chao. 1977. Resource-limited growth, competition, and predation: a model and experimental studies with bacteria and bacteriophage. American Naturalist 111:3-24.

Levin, S. A., D. Cohen, and A. Hastings. 1984. Dispersal strategies in patchy environments. Theoretical Population Biology 26:156-191.

Levins, R. 1979. Coexistence in a variable environment. American Naturalist 114:765-783.

Ludwig, D., and S. A. Levin. 1991. Evolutionary stability of plant communities and the maintenance of multiple dispersal types. Theoretical Population Biology 40:285-307.

Maynard Smith, J. 1982. Evolution and the theory of games. Cambridge University Press, Cambridge.

Nipkow, F. 1961. Die Radertiere im Plankton des Zurichsees und ihre Entwicklungsphasen. Schweizerische Zeitschrift für Hydrobiologie 23:398-461.

Philippi, T., and J. Seger. 1989. Hedging one's evolutionary bets, revisited. Trends in Ecology \& Evolution 4:41-44.

Sandgren, C. D. 1988. Growth and reproduction strategies in freshwater phytoplankton. Cambridge University Press, New York.

Seger, J., and H. J. Brockmann. 1987. What is bet-hedging? Oxford Surveys in Evolutionary Biology 4:182-211.

Shmida, A., and S. Ellner. 1984. Coexistence of plant species with similar niches. Vegetatio 58:29-55.

Slatkin, M. 1974. Hedging one's evolutionary bets. Nature (London) 250:704-705.

Tauber, M. J., C. A. Tauber, and S. Masaki. 1986. Seasonal adaptations of insects. Oxford University Press, New York.

Templeton, A. R., and D. A. Levin. 1979. Evolutionary consequences of seed pools. American Naturalist 114:232-249.

Turelli, M. 1978. A reexamination of stability in randomly varying versus deterministic environments with comments on the theory of limiting similarity. Theoretical Population Biology 13: 244-267.

1988. Population genetic models for polygenic variation and evolution. Pages 601-618 in E. J. Eisen, M. M. Goodman, G. Namkoong, and B. S. Weir, eds. The Second International Conference on Quantitative Genetics. Sinauer, Boston.

Venable, D. L. 1989. Modeling the evolutionary ecology of seed banks. Pages 67-90 in M. A. Leck, V. T. Parker, and R. L. Simpson, eds. Ecology of soil seed banks. Academic Press, New York.

Warner, R. R., and P. L. Chesson. 1985. Coexistence mediated by recruitment fluctuations: a field guide to the storage effect. American Naturalist 125:769-787. 